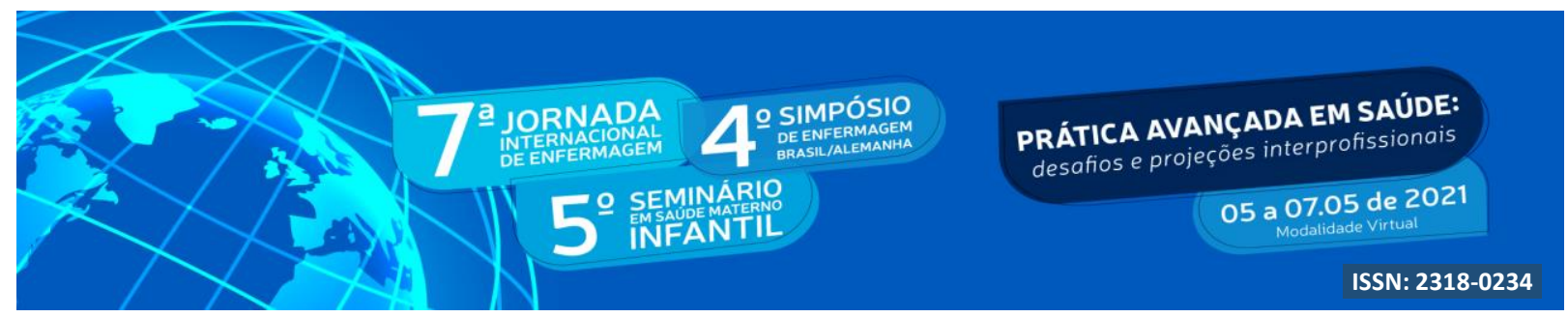

DOI: http://doi.org/10.48195/jie2021-108

\title{
PANORAMA ATUAL DOS EVENTOS ADVERSOS RELACIONADOS À ASSISTÊNCIA À SAÚDE: REVISÃO INTEGRATIVA DE LITERATURA
}

\author{
Eliete Aparecida Teodoro Amaral'; Eliane Ramos Pereira²; Janaína Mengal Gomes \\ Fabri $^{3}$
}

\begin{abstract}
RESUMO
Introdução: A prevenção de Eventos adversos (EA) é um tópico primordial para a qualidade da assistência em saúde. Objetivo: Refletir sobre o panorama atual das ações estratégicas de minimização da ocorrência de Eventos Adversos relacionados à Assistência à saúde. Método: As buscas foram realizadas no Portal Periódicos Capes pelos descritores Eventos Adversos; Controle de Risco e Segurança do Paciente. Resultados e Discussão: Foram considerados para análise 33 artigos. Observou-se citações acerca de instrumentos tais como os checklists, o Trigger Tool, mnemônicos e a própria tecnologia da informação. Correlacionam-se possíveis fatores que concorrem para a ocorrência de EA tais como a carga horária de trabalho e a internalização da cultura de segurança. Conclusão: No panorama atual, há uma preocupação contínua com a segurança e qualidade da assistência e o desenvolvimento de ferramentas e estratégias que viabilizem a assistência segura são fundamentais.
\end{abstract}

Palavras-chave: Controle de Risco; Eventos Adversos; Segurança do Paciente.

\begin{abstract}
Introduction: The prevention of adverse events (AE) is a major issue for the quality of health care. Objective: To reflect on the current panorama of strategic actions to minimize the occurrence of Adverse Events related to Health. Method: As you search for forms, you do not make Portal Jornais Capes by the descriptors Adverse Events; Risk Control and Patient Safety. Results and Discussion: 33 articles were considered for analysis. Observations are made on instruments such as checklists, or Trigger Tool, mnemonics and proprietary information technology. We correlate with factors that correspond to the occurrence of AE, such as the workload and the internalization of the safety culture. Conclusion: Without current perspectives, the concern with the guarantee and quality of assistance persists and / or the development of tools and strategies that enable safe and grounded assistance.
\end{abstract}

Key Words: Risk Management; Medical Error; Pacient Safety Care.

\footnotetext{
${ }^{1}$ Mestranda do Programa de Mestrado Profissional de Enfermagem Assistencial - MPEA/UFF. Universidade Federal Fluminense. E-mail: elieteaparecida@gmail.com

${ }^{2}$ Professora Titular da EEAAC/ Universidade Federal Fluminense/ UFF. Pós-Doutorado pela Universidade do Estado do Rio de Janeiro/ UERJ. Universidade Federal Fluminense. E-mail: elianeramos.uff@ gmail.com

${ }^{3}$ Doutoranda do Programa Acadêmico Ciências do Cuidado e Saúde - PACCS/UFF. Universidade Federal Fluminense. E-mail: janamgfabri@gmail.com
} 


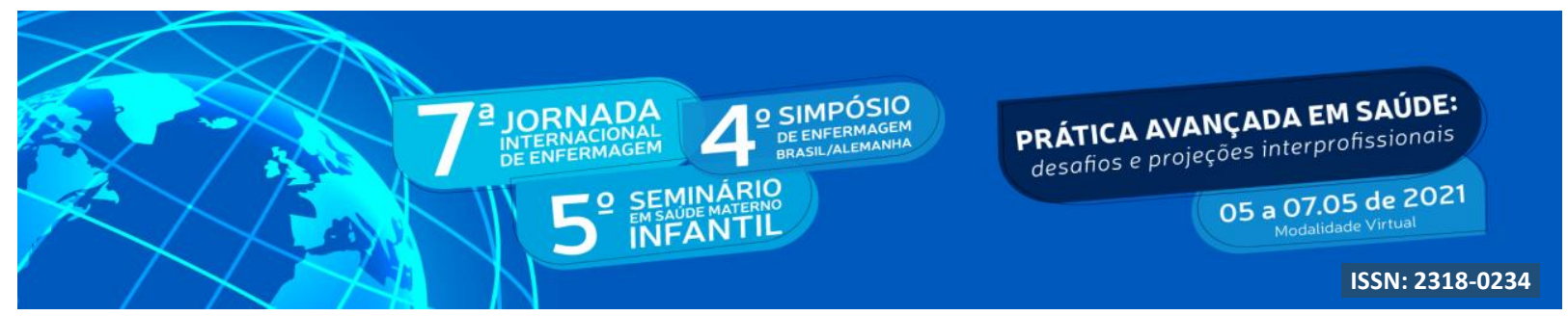

\section{INTRODUÇÃO}

Eventos adversos (EA) durante a assistência à saúde tem se tornado pauta recorrentes nos diversos cenários de saúde. O último dado estatístico relacionado a incidentes relacionados à assistência à saúde datam do ano de 2016, e divulgam-se um total de 53.997 EA no ano indicado, sendo estes eventos em sua maioria falhas durante a assistência à saúde (14.068 ocorrências), lesões por pressão (10.210 ocorrências) e quedas do paciente (5.892 ocorrências). (ANVISA, 2017)Tendo como base esses pontos críticos, muitas ações foram implementadas, desde então, no intuito de diminuir a ocorrência de eventos adversos na assistência à saúde, em vários cenários e em múltiplos prismas.

Dentro dessa perspectiva, questiona-se quais as ações desenvolvidas a partir das publicações acerca das diretrizes da assistência segura até os dias atuais, que visaram ou visam a diminuição ou eliminações da ocorrência de Eventos Adversos.

\section{OBJETIVO}

Refletir sobre o panorama atual das ações estratégicas de minimização da ocorrência de Eventos Adversos relacionados à Assistência à saúde

\section{METODOLOGIA}

Este estudo tratou-se de uma revisão integrativa de literatura, que tem como principal finalidade "sintetizar resultados obtidos em pesquisas sobre um tema ou questão, de maneira sistemática, ordenada e abrangente. Foram realizadas buscas no Portal Periódicos Capes pelos descritores Eventos Adversos; Controle de Risco e Segurança do Paciente. O Portal foi escolhido por possibilitar de modo gratuito acesso a inúmeros periódicos e publicações científicas. Os critérios de inclusão foram estudos que apresentassem reflexões, métodos, ferramentas ou instrumentos que viabilizassem a minimização da ocorrência de Eventos Adversos relacionados à Assistência à saúde. Foram aplicados os filtros: lapso temporal de 2014 a 2019 - justificado por ser o ano posterior às publicações contendo diretrizes e definições sobre os eventos adversos e as ações preconizadas para minimizá-los; e idioma, sendo o português a língua priorizada para análise.

A exclusão se deu a estudos que não se referissem às atividades relacionadas a 


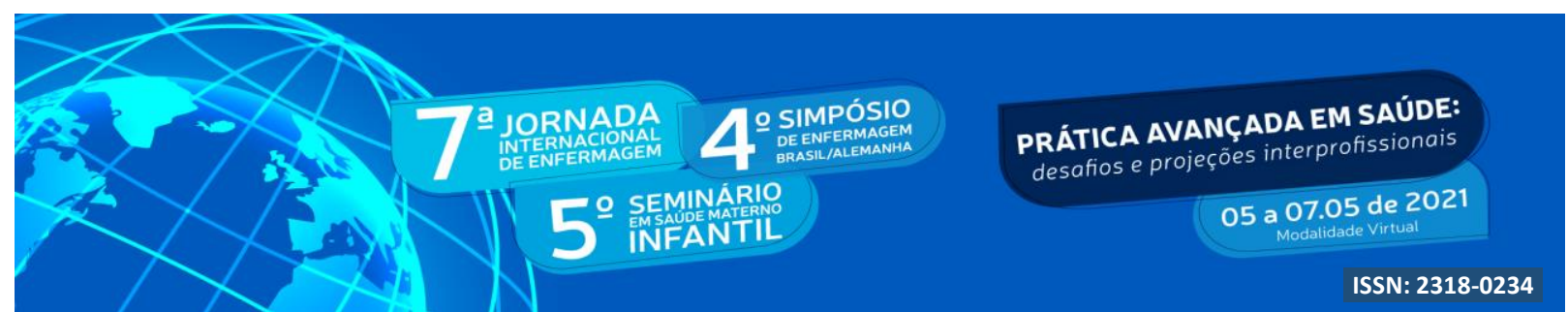

Segurança do Paciente realizadas no Brasil. Aplicados os critérios de inclusão e exclusão, e os filtros supracitados, foram encontrados 161 artigos. Após análise preliminar de resumos, foram triados 36 artigos. A leitura dos artigos gerou um total de 33 produções que atendiam à temática. (Tabela 1)

Tabela 1 - Resultados de Busca e Seleção dos Estudos.

\section{Portal de Periódicos Capes}

Análise de Títulos Análise de Resumos Análise de Artigos

\begin{tabular}{cccccc}
\hline Total & Selecionados & Total & Selecionados & Total & Selecionados \\
\hline $\mathbf{1 6 1}$ & 39 & 39 & 36 & 36 & 33 \\
\hline
\end{tabular}

Fonte: Busca realizado pelo autor no periódico Capes. 2019.

\section{RESULTADOS E DISCUSSÃO}

Os 33 artigos selecionados foram dispostos na tabela a seguir, apresentando-se os principais dados encontrados:

Tabela 2 - Apresentação dos artigos selecionados para a revisão de literatura.

\begin{tabular}{|c|c|c|c|}
\hline & Estudo & Autores & Ano \\
\hline 1 & Eventos Adversos: estratégias e incentivos para notificações hospitalares. & $\begin{array}{l}\text { MOREIRA, A.A.O. } \\
\text { REMONDI, G.C. } \\
\text { ORTEGA, M.C.C. } \\
\text { VERLINGUE, S. } \\
\text { ORTEGA, N.N.C. }\end{array}$ & 2018 \\
\hline 2 & Mudanças na Prática de Enfermagem para Melhorar a segurança do paciente. & $\begin{array}{l}\text { SIMAN, A.G. } \\
\text { BRITO, M.J.M. }\end{array}$ & 2016 \\
\hline 3 & $\begin{array}{l}\text { Segurança do Paciente em Unidades de Terapia Intensiva: desenvolvimento de um } \\
\text { projeto de pesquisa. }\end{array}$ & $\begin{array}{l}\text { PADILHA, K.G. } \\
\text { BARBOSA, R.L. } \\
\text { OLIVEIRA, E.M. } \\
\text { ANDOLHE, R. } \\
\text { DUCCI, A.J. } \\
\text { SECOLI, S.R. }\end{array}$ & 2015 \\
\hline 4 & $\begin{array}{l}\text { Estratégias para a Segurança do Paciente no Processo de Uso de Medicamentos } \\
\text { Após Alta Hospitalar. }\end{array}$ & $\begin{array}{l}\text { MARQUES, L.F.G. } \\
\text { ROMANO-LIEBER, N.S. }\end{array}$ & 2014 \\
\hline 5 & $\begin{array}{l}\text { Banho no Leito: carga de trabalho da equipe de enfermagem e segurança do } \\
\text { paciente. }\end{array}$ & $\begin{array}{l}\text { MOLLER, G. } \\
\text { MAGALHÃES, A.M.M. }\end{array}$ & 2015 \\
\hline 6 & $\begin{array}{l}\text { Análise Normativa dos Comitês de Investigação de Óbitos, de Controle de Infecção } \\
\text { e sua Interface com a Segurança do Paciente em Organizações Complexas. }\end{array}$ & ALVES, E.A.V. & 2015 \\
\hline 7 & Proposta de um Instrumento para Avaliar Eventos Adversos em Odontologia. & $\begin{array}{l}\text { MENDES, W. } \\
\text { CORRÊA, C.D.T.S.O. }\end{array}$ & 2017 \\
\hline
\end{tabular}




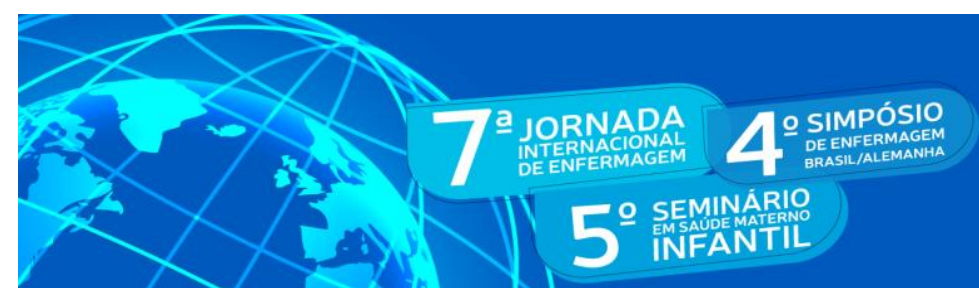

PRÁTICA AVANÇADA EM SAÚdE desafios e projeçôes interprofissionais

05 a 07.05 de 2021

05 a 07.05 didade Virtua

ISSN: 2318-0234

Eventos Adversos em Unidades de Terapia Intensiva: estudo bibliométrico.

Tipos de Gestão.

11 Sobrecarga de Trabalho da Enfermagem e Incidentes e Eventos Adversos em Pacientes Internados em UTI.

Tecnologias na Terapia Intensiva: causas dos eventos adversos e implicações para a enfermagem.

Tecnovigilância no Brasil: panorama das notificações de eventos adversos e queixas técnicas de cateteres vasculares.

Características dos Eventos Adversos na Atenção Primária à Saúde no Brasil.

Eventos Adversos Identificados nos Relatórios de Enfermagem em uma Clínica Pediátrica.

Clima de Segurança do Paciente em um Hospital de Ensino.

Comunicação Efetiva para a Segurança do Paciente: nota de transferência e Modified Early Warning Score.

Trigger Tool na Segurança do Doente: uma revisão sistemática de literatura.

Identificação do Paciente por Pulseira Eletrônica numa Unidade de Terapia Intensiva Geral Adulta.

Notificação de Eventos Adversos: caracterização dos eventos ocorridos em um complexo hospitalar.

Avaliação da Adesão à Lista de Verificação de Segurança no Parto em uma Maternidade Pública no Nordeste do Brasil.

Avaliação da Adesão ao Checklist de Cirurgia Segura da OMS em Cirurgias Urológicas e Ginecológicas, em Dois Hospitais de Ensino de Natal, Rio Grande do Norte, Brasil.

Processos Desenvolvidos por Gestores de Enfermagem Face ao Erro.

Percepção dos Profissionais de Saúde Sobre a Cultura de Segurança do Doente Pediátrico.

DUARTE, S.C.M.

STIPP, M.A.C.

SILVA, M.M.

OLIVEIRA, F.T.

DUTRA, D.D.

DUARTE, M.C.S.

ALBUQUERQUE, K.F.

SANTOS, J.S

SIMÕES, K.M

ARARUNA, P.C.

ANDRADE, L.E.L.

Et. al.

NOVARETTI, M.C.Z.

SANTOS, E.V.

QUITÉRIO, L.M.

DAUD-GALLOTTI, R.M

RIBEIRO, G.S.R.

SILVA, R.C.

FERREIRA, M.A.

OLIVEIRA, C.G.

RODAS, A.C.D.

MARCHON, S.G.

MENDES JUNIOR, W.V.

PAVÃO, A.L.B.

ROCHA, J.P.

SILVA, A.E.B.C.

BEZERRA, A.L.Q

SOUSA, M.R.G.

MOREIRA, I.A.

MAGALHÃES, F.H.L

PEREIRA, I.C.A.

LUIZ, R.B.

BARBOSA, M.H.

FERREIRA, M.B.G.

OLINO, L.

PIERDEVARA, L.

VENTURA, I.M.

EIRAS, M.

GRACIA, A.M.B.

MACEDO, M.C.S

ALMEIDA, L.F.

ASSAD, L.G.

ROCHA, R.G.

RIBEIRO, G.S.R.

PEREIRA, L.M.V.

FURINI, A.C.A.

NUNES, A.A.

DALLORA, M.E.L.V.

PRAXEDES, A.O

ARRAIAS, L.

ARAÚJO, M.A.A

SILVA, E.M.M.

GAMA, Z.A.S.

FREITAS, M.R.

FREITAS, M.R.

ANTUNES, A.G.

LOPES, B.N.A.

FERNANDES, F.C.

MONTE, L.C.

GAMA, Z.A.S

CORREIA, T.S.P.

MARTINS, M.M.F.P.S.

2014

FORTE, E.C.N.

SILVA, E.M.B.

PEDROSA, D.L.L.

LECA, A.P.C.

SILVA, D.M. 


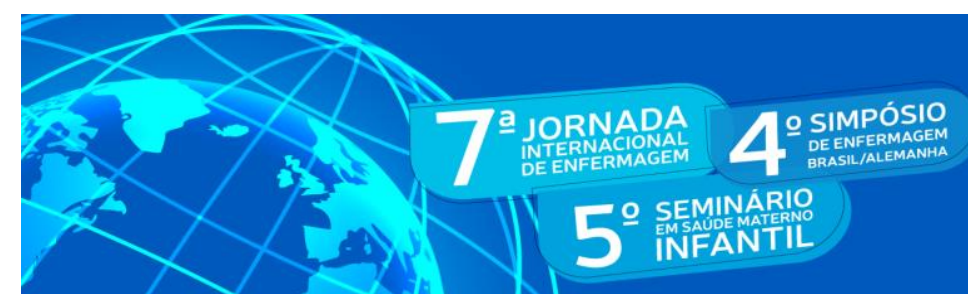

PRÁTICA AVANÇADA EM SAÚDE:

desafios e projeções

05 a 07.05 de 2021

ISSN: 2318-0234

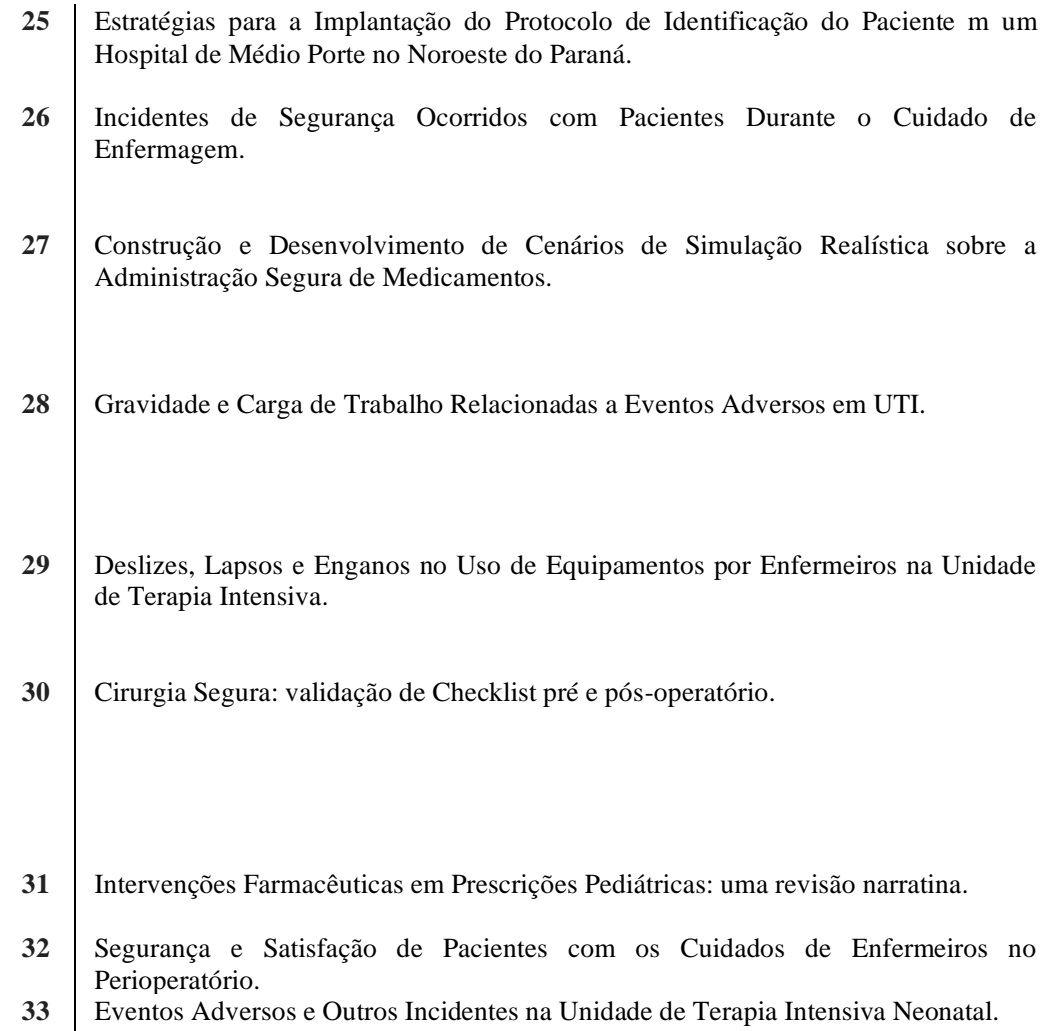

REBELLO, L.K.Z.

2019

QUERNEL, F.S.

PETERLINI, O.L.G.

HOEFEL, H.H.K.

LUCENA, A.F.

MANTOVANI, V.M.

COGO, A.L.P.

LOPES, E.F.S.

PERDOMINI, F.R.I.

FLORES, G.E.

SANTOS, M.R.R.

SERAFIM, C.T.R.

DELL'ACQUA, M.C.Q

CASTRO, M.C.N.

SPIRI, W.C.

NUNES, H.R.C

RIBEIRO, G.S.R.

SILVA, R.C.

FERREIRA, M.A.

SILVA, G.R.

ALPENDRE, F.T.

CRUZ, E.D.A.

DYNIEWICZ, A.M

MANTOVANI, M.F

SILVA, A.E.B.C.

SANTOS, G.S

BECKER, G.C.

BUENO, D.

SILLERO-SILLERO, A. 2019

ZABALEGUI, A.

LANZILLOTTI, L.S.

2015

SETA, M.H.

ANDRADE, C.L.T.

MENDES JUNIOR, W.V.

2019

2017

2016

Fonte: Busca realizado pelo autor no periódico Capes. 2020.

\section{Identificação e Diminuição do Risco de Eventos Adversos}

O Brasil possui uma grande incidência de eventos adversos, no ambiente hospitalar essa incidência varia de 10 a $60 \%$ dos pacientes. É preciso evidenciar que diversos fatores influenciam esta incidência, principalmente o que diz respeito a estrutura física das unidades, a tecnologia disponível na unidade e o contingente adequado ou não de profissionais prestando assistência. (NAVARETTI, 2014; MOLLER, 2015; RIBEIRO, 2016)

Outro fator de grande influência é a equipe de enfermagem, maior força de trabalho nas unidades de saúde, e fatores como carga de trabalho, estresse, satisfação dos profissionais, salários, características do serviço e o ambiente de segurança institucional. Os eventos adversos em suas ocorrências em geral apresentam uma gravidade alarmante, o estudo de MARCHON (2015) que cerca de $26 \%$ dos pacientes que sofrem danos estes chegam a se 


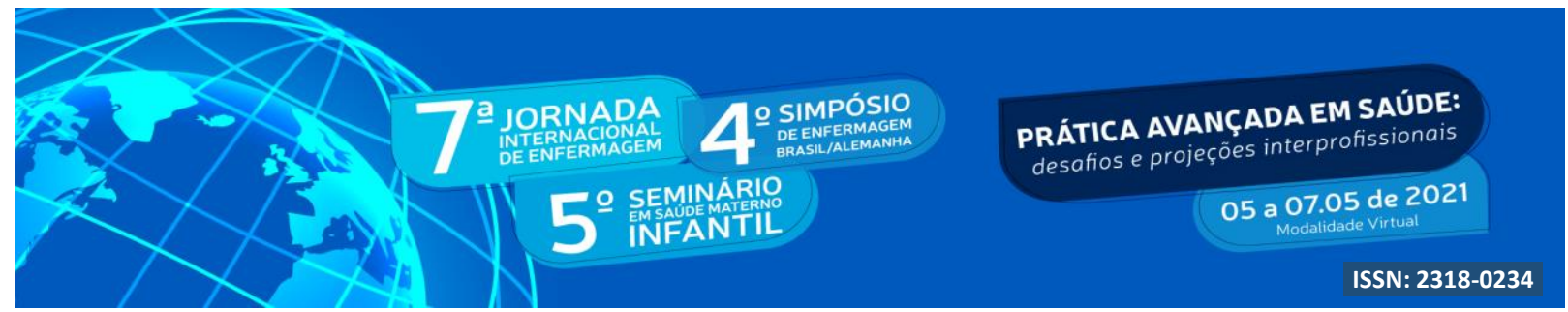

importância da equipe de enfermagem atuar para prevenir eventos adversos. (PADILHA, 2015; DUTRA, 2017)

A prevenção destes eventos é fundamental, já que a ocorrências destas falhas é utilizada na maioria das unidades como indicador de qualidade da assistência. Desta forma é fundamental diminuir a incidência destes erros e, assim, não comprometer a segurança do paciente e os indicadores de qualidade das instituições de saúde, e para que isso ocorra é preciso que haja a gestão de riscos, dando oportunidade para a instituição lidar com os erros. (SERAFIM, 2017; CORREIA, 2017)

Não somente a qualidade da assistência e os indicadores se veem afetados, os erros de assistência são responsáveis pelo aumento dos custos das instituições de saúde, geram sofrimentos no paciente e aumentam o período de permanência do paciente nos serviços hospitalares. Portanto, é necessária a adoção de protocolos institucionais e a adoção de medidas de segurança para prevenir situações de risco e promover uma melhoria na segurança do paciente. (LANZILLOTTI, 2015; OLINO, 2019)

\section{Notificação de Eventos Adversos}

A análise das notificações permite a aprendizagem organizacional, possibilitando que as causas sejam identificadas e evitadas" (FURINI, 2019, p. 2).

Entretanto, identifica-se uma grande resistência por parte dos profissionais em relatar a ocorrência de eventos adversos, e isso dificulta que os gestores possam identificar as falhas da assistência da unidade e criar medidas ou barreiras para melhoria da assistência da unidade (PIERDEVARA, 2017; OLIVEIRA, 2017). E uma forma de melhorar isso é incentivar que os profissionais notifiquem estas ocorrências através de sistemas informativos, que interliguem as "ações a fim de detectar e analisar eventos adversos e situações de risco, além de auxiliar no direcionamento de quais atividades educacionais devem ser desenvolvidas para melhorar a segurança de pacientes" (MOREIRA, 2018, p. 102).

\section{Cultura de Segurança do Paciente e Melhoria da Qualidade da Assistência}




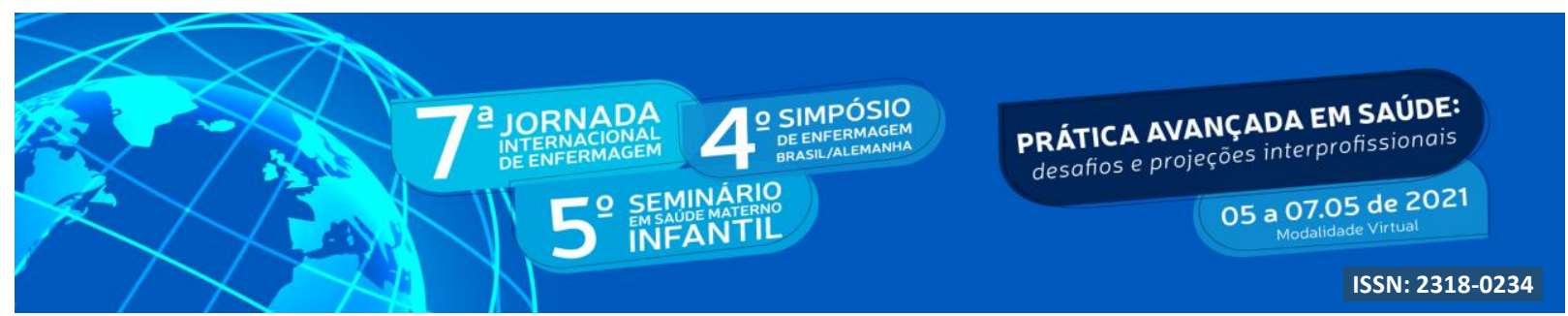

É fundamental definir o que vem a ser a cultura de segurança do paciente, e esta é definida como:

A cultura de segurança de qualquer organização é um fenómeno complexo, de caráter multidimensional. Tendo em conta a abrangência do conceito, não existe consenso quanto às dimensões que integra e os aspetos a considerar para o seu desenvolvimento. (SILVA, 2016, p. 88)

A cultura de segurança é um compromisso institucional, de todos os profissionais da unidade e que busca promover ações segura aos pacientes. Para isso a transmissão de valores e objetivos de segurança e qualidade da assistência é fundamental para se estabelecer a cultura de segurança nas instituições. (MAGALHÃES, 2019; SILLERO-SILLERO, 2019)

\section{Estratégias Específicas de Prevenção à Eventos Adversos}

Algumas áreas específicas são de vital importância para a segurança do paciente, uma delas são os medicamentos, na questão os indicadores $19 \%$ dos eventos adversos estão relacionados a medicação. Essas ocorrências representam uma causa importante de morbimortalidade associada a falhas da assistência. E no contexto do ambiente hospitalar uma pareceria que pode ser muito benéfica é a do enfermeiro com o farmacêutico clínico, esses profissionais podem trabalhar em equipe para criar estratégias que visem reduzir a ocorrência de erros no uso de medicamentos. (MARQUES, 2014; COGO, 2019)

Uma estratégia muito efetiva é educar os profissionais, existem diversas técnicas que visam promover a melhoria da segurança e ensinar aos profissionais essas técnicas pode melhorar muito os índices de ocorrência destes eventos adversos. (COGO, 2019)

Unidades que são de existência obrigatória atualmente nas unidades hospitalares, e visa a garantia da segurança do paciente; identificação, controle e diminuição de eventos adversos; e o controle e prevenção de infecções. Os profissionais atuantes nestes setores têm um papel fundamental na questão da cultura do paciente, e melhoria da qualidade da assistência. (ALVES, 2015)

\section{CONCLUSÃO}

No panorama atual, há uma preocupação contínua com a segurança e qualidade da 


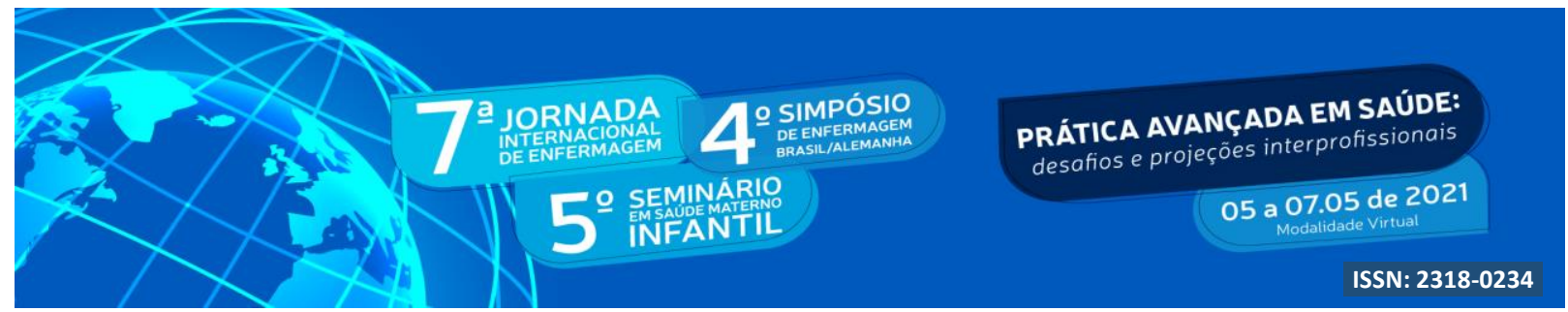

assistência e o desenvolvimento de ferramentas e estratégias que viabilizem a assistência segura são fundamentais.

\section{REFERÊNCIAS}

MOREIRA, A.A.O.; REMONDI, G.C.; ORTEGA, M.C.C.; VERLINGUE, S.; ORTEGA, N.N.C. Eventos Adversos: estratégias e incentivos para notificações hospitalares. R. Saúde Publ. Paraná, v. 1, n. 1, p. 101-107, Jul. 2018.

SIMAN, A.G.; BRITO, M.J.M. Mudanças na Prática de Enfermagem para Melhorar a segurança do paciente. Rev. Gaúcha Enferm., v. 37, n. esp. 2016.

PADILHA, K.G. ; BARBOSA, R.L.; OLIVEIRA, E.M.; ANDOLHE, R.; DUCCI, A.J.; SECOLI, S.R. Segurança do Paciente em Unidades de Terapia Intensiva: desenvolvimento de um projeto de pesquisa. Rev. Esc. Enferm. USP, v. 49, n. esp., p. 157-163. 2015.

MARQUES, L.F.G.; ROMANO-LIEBER, N.S. Estratégias para a Segurança do Paciente no Processo de Uso de Medicamentos Após Alta Hospitalar. Physis Rev. de Saúde Col., v. 24, n. 2, p. 401-420. 2014.

MOLLER, G.; MAGALHÃES, A.M.M. Banho no Leito: carga de trabalho da equipe de enfermagem e segurança do paciente. Texto Cont. Enferm., v. 24, n. 4, p. 1044-1052, Out-Dez. 2015.

ALVES, E.A.V. Análise Normativa dos Comitês de Investigação de Óbitos, de Controle de Infecção e sua Interface com a Segurança do Paciente em Organizações Complexas. Cad. IberoAmer. Dir. Sanit., v. 4, n. 4, Out-Dez. 2015.

MENDES, W.; CORREAA, C.D.T.S.O. Proposta de um Instrumento para Avaliar Eventos Adversos em Odontologia. Cad. Saúde Públ., v. 33, n. 11. 2017.

DUARTE, S.C.M.; STIPP, M.A.C.; SILVA, M.M.; OLIVEIRA, F.T. Eventos Adversos e Segurança na Assistência de Enfermagem. Rev. Bras. Enferm., v. 68, n. 1, Jan-Fev. 2015.

DUTRA, D.D.; DUARTE, M.C.S.; ALBUQUERQUE，K.F.; SANTOS， J.S.; SIMÕES， K.M.; ARARUNA, P.C. Eventos Adversos em Unidades de Terapia Intensiva: estudo bibliométrico. J. Res. Fundam. Care., v. 9, n. 3, Jul-Set. 2017.

ANDRADE, L.E.L.; Et. al. Cultura de Segurança do Paciente em Três Hospitais Brasileiros com Diferentes Tipos de Gestão. Ciência \& Saú. Col., v. 23, n. 1, p. 161-172. 2018.

NOVARETTI, M.C.Z.; SANTOS, E.V.; QUITÉRIO, L.M.; DAUD-GALLOTTI, R.M. Sobrecarga de Trabalho da Enfermagem e Incidentes e Eventos Adversos em Pacientes Internados em UTI. Rev. Bras. Enferm., v. 67, n. 5, p. 692-699, Set-Out. 2014.

RIBEIRO, G.S.R.; SILVA, R.C.; FERREIRA, M.A. Tecnologias na Terapia Intensiva: causas dos eventos adversos e implicações para a enfermagem. Rev. Bras. Enferm., v. 69, n. 5, Set-Out. 2016. 


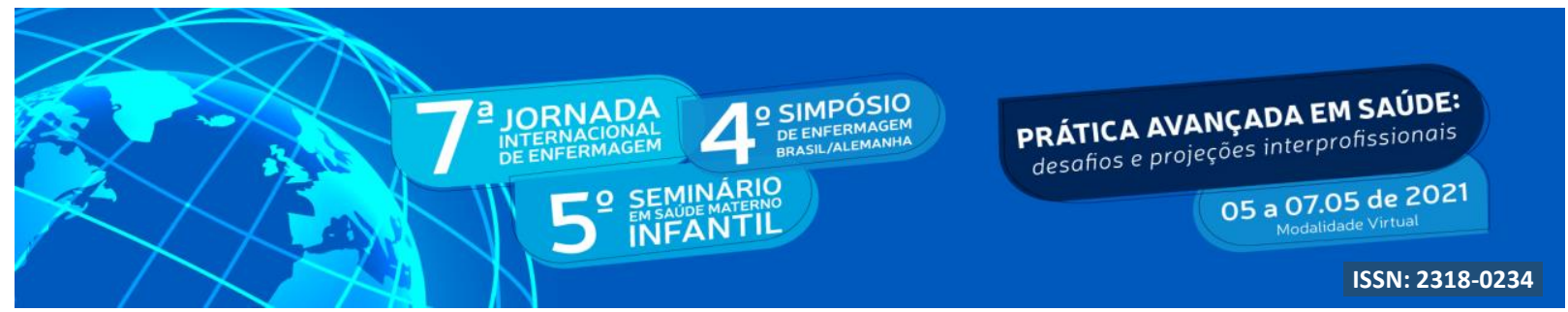

OLIVEIRA, C.G.; RODAS, A.C.D. Tecnovigilância no Brasil: panorama das notificações de eventos adversos e queixas técnicas de cateteres vasculares. Ciência \& Saú. Col., v. 22, n. 10, p. 3247-3257. 2017.

MARCHON, S.G.; MENDES JUNIOR, W.V.; PAVÃO, A.L.B. Características dos Eventos Adversos na Atenção Primária à Saúde no Brasil. Cad. Saúde Publ., v. 31, n. 11, p. 2313-2330, Nov. 2015.

ROCHA, J.P.; SILVA, A.E.B.C.; BEZERRA, A.L.Q.; SOUSA, M.R.G.; MOREIRA, I.A. Eventos Adversos Identificados nos Relatórios de Enfermagem em uma Clínica Pediátrica. Ciên. y Enferm., v. 20, n. 2, p. 53-63. 2014.

MAGALHÃES, F.H.L.; PEREIRA, I.C.A.; LUIZ, R.B.; BARBOSA, M.H.; FERREIRA, M.B.G. Clima de Segurança do Paciente em um Hospital de Ensino. Rev. Gaúcha Enferm., v. 40, n. esp. 2019.

OLINO, L.; Et. al. Comunicação Efetiva para a Segurança do Paciente: nota de transferência e Modified Early Warning Score. Rev. Gaúcha Enferm., v. 40, n. esp. 2019.

PIERDEVARA, L.; VENTURA, I.M.; EIRAS, M.; GRACIA, A.M.B. Trigger Tool na Segurança do Doente: uma revisão sistemática de literatura. Port. J. Public. Health, v. 35, p. 69-76.2017.

MACEDO, M.C.S.; ALMEIDA, L.F.; ASSAD, L.G.; ROCHA, R.G.; RIBEIRO, G.S.R.; PEREIRA, L.M.V. Identificação do Paciente por Pulseira Eletrônica numa Unidade de Terapia Intensiva Geral Adulta. Rev. Enferm. Referência, v. 4, n. 13, p. 63-70, Abr.-Jun. 2017.

FURINI, A.C.A.; NUNES, A.A.; DALlORA, M.E.L.V. Notificação de Eventos Adversos: caracterização dos eventos ocorridos em um complexo hospitalar. Rev. Gaúcha Enferm., v. 40, n. esp. 2019.

PRAXEDES, A.O.; ARRAIAS, L.; ARAÚJO, M.A.A.; SILVA, E.M.M.; GAMA, Z.A.S.; FREITAS, M.R. Avaliação da Adesão à Lista de Verificação de Segurança no Parto em uma Maternidade Pública no Nordeste do Brasil. Cad. Saúde Publ., v. 33, n. 10. 2017.

FREITAS, M.R.; ANTUNES, A.G.; LOPES, B.N.A.; FERNANDES, F.C.; MONTE, L.C.; GAMA, Z.A.S. Avaliação da Adesão ao Checklist de Cirurgia Segura da OMS em Cirurgias Urológicas e Ginecológicas, em Dois Hospitais de Ensino de Natal, Rio Grande do Norte, Brasil. Cad. Saúde Publ., v. 30, n. 1, p. 137-148, Jan. 2014.

CORREIA, T.S.P.; MARTINS, M.M.F.P.S.; FORTE, E.C.N. Processos Desenvolvidos por Gestores de Enfermagem Face ao Erro. Rev. Enferm. Referência, v. 4, n. 12, p. 75-84, Jan.-Mar. 2017.

SILVA, E.M.B.; PEDROSA, D.L.L.; LEÇA, A.P.C.; SILVA, D.M. Percepção dos Profissionais de Saúde Sobre a Cultura de Segurança do Doente Pediátrico. Rev. Enferm. Referência, v. 4, n. 9, p. 87-95, Abr.-Jun. 2016.

REBELLO, L.K.Z.; QUERNEL, F.S.; PETERLINI, O.L.G. Estratégias para a Implantação do Protocolo de Identificação do Paciente m um Hospital de Médio Porte no Noroeste do Paraná. R. Saúde Publ. Paraná, v. 2, n. 1, p. 31-37, Jul. 2019. 


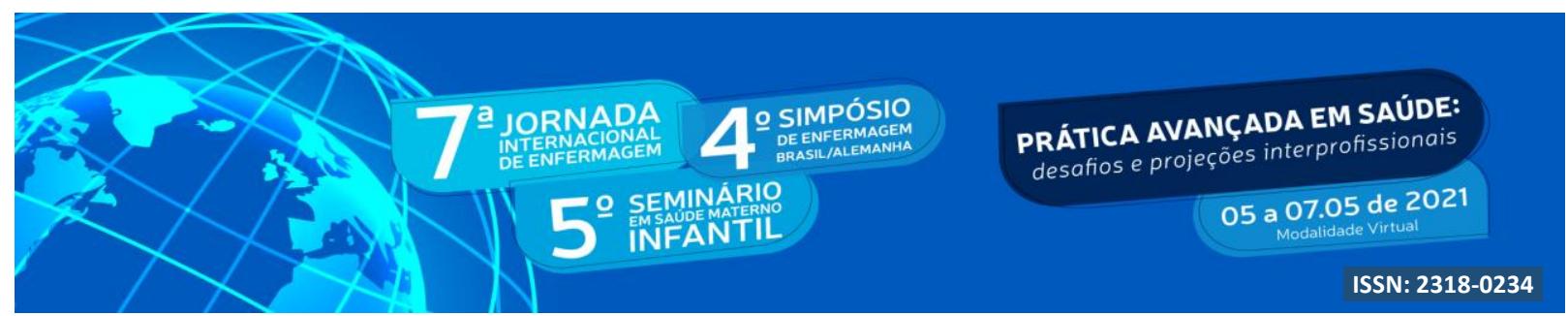

HOEFEL, H.H.K.; ECHER, I.; LUCENA, A.F.; MANTOVANI, V.M. Incidentes de Segurança Ocorridos com Pacientes Durante o Cuidado de Enfermagem. R. Epidemiol. Control. Infec., v. 7 , n. 3, p. 174-180. 2017.

COGO, A.L.P.; LOPES, E.F.S.; PERDOMINI, F.R.I.; FLORES, G.E.; SANTOS, M.R.R. Construção e Desenvolvimento de Cenários de Simulação Realística sobre a Administração Segura de Medicamentos. Rev. Gaúcha Enferm., v. 40, n. esp. 2019.

SERAFIM, C.T.R.; DELL'ACQUA, M.C.Q.; CASTRO, M.C.N.; SPIRI, W.C.; NUNES, H.R.C. Gravidade e Carga de Trabalho Relacionadas a Eventos Adversos em UTI. Rev. Bras. Enferm., v. 70, n. 5, p. 993-999. 2017.

RIBEIRO, G.S.R.; SILVA, R.C.; FERREIRA, M.A.; SILVA, G.R. Deslizes, Lapsos e Enganos no Uso de Equipamentos por Enfermeiros na Unidade de Terapia Intensiva. Rev. Esc. Enferm. USP, v. 50, n. 3, p. 419-426. 2016.

ALPENDRE, F.T.; CRUZ, E.D.A.; DYNIEWICZ, A.M.; MANTOVANI, M.F.; SILVA, A.E.B.C.; SANTOS, G.S. Cirurgia Segura: validação de Checklist pré e pós-operatório. Rev. Latino-Am. Enferm., v. 25, n. esp. 2017.

BECKER, G.C.; BUENO, D. Intervenções Farmacêuticas em Prescrições Pediátricas: uma revisão narratina. Clin. Biomed. Res., v. 38, n. 4., p. 396-402. 2018.

SIllERO-SILlERO, A.; ZABALEGUI, A. Segurança e Satisfação de Pacientes com os Cuidados de Enfermeiros no Perioperatório. Rev. Latino-Am. Enferm., v. 27, n. esp. 2019.

LANZILlOTTI, L.S.; SETA, M.H.; ANDRADE, C.L.T.; MENDES JUNIOR, W.V. Eventos Adversos e Outros Incidentes na Unidade de Terapia Intensiva Neonatal. Ciênc. \& Saúde Col., v. 20, n. 3, p. 937-946. 2015.

MINISTÉRIO DA SAÚDE. Documento de Referência para o Programa Nacional de Segurança do Paciente. Brasília: O Ministério, 2014.

ANVISA - Agência Nacional de Vigilância Sanitária. Boletim de Segurança do Paciente e Qualidade em Serviços de Saúde no 15: incidentes relacionados à assistência à saúde - 2016. Brasília, 2017. Disponível em: < portal.anvisa.gov.br>.

MESQUITA, K.O.; SILVA, L.C.C.; LIRA, R.C.M.; FREITAS, C.A.S.L.; LIRA, G.V. Segurança do Paciente na Atenção Primária à Saúde: revisão integrativa. Cogitare Enfermagem, v. 21, n. 2, p. 18, abr/jun. 2016.

GOMES, A.T.L.; et. al. A Segurança do Paciente nos Caminhos Percorridos pela Enfermagem Brasileira. Rev. Bras. de Enfermagem, v. 70, n. 1, p. 146-54, jan-fev. 2017.

ALVES, M.F.T.; CARVALHO, D.S.; ALBUQUERQUE, G.S.C. Motivos para Não Notificação de Incidentes de Segurança do Paciente por Profissionais de Saúde: revisão integrativa. Cienc. E Saúde Coletiva, v. 24, n. 8, p. 2895-908. 2019. 